\title{
La fédération des entreprises du Congo : organisation, fonctionnement et compétences
}

\author{
Par Marc Kamananga Mangaza*
}

\begin{abstract}
Résumé
La RDC est un pays dont le potentiel économique n'est plus à démontrer. Les acteurs privés impliqué dans cette dynamiques au niveau national ont pris la décision de se regrouper de leur propre initiative au tour de la Fédération des entreprises du Congo (FEC), qui est une asbl aux termes de la loi $\mathrm{n}^{\circ} 004 / 2001$ du 20juillet 2001 portant dispositions générales applicables aux associations sans but lucratif et aux établissements d'utilité publique. Cette institution privé permet aux entrepreneurs congolais de se réunir et de discuter à propos des sujets qui intéressent leurs activités et, le cas échéant, défendre leurs intérêts pour la réalisation de leurs objectifs individuels ou collectifs. La FEC dispose des organes nationaux et des organes provinciaux et municipaux. Ainsi, elle comprendre des organes à compétence nationale générale qui sont l'Assemblée Générale; le Conseil d'Administration; le Comité de Direction et les Commissaires aux comptes); mais également des organes à compétence nationale spéciale dénommés Commissions ou Chambres et des organes à compétence matérielle générale dans les limites de leur ressort territorial qui sont l'Assemblée Provinciale; le Conseil Provincial; le Comité de District; le Comité de Territoire ou de Commune.
\end{abstract}

\begin{abstract}
The DRC is a country whose economic potential is no longer to be demonstrated. The private actors involved in this dynamic at the national level have taken the decision to regroup on their own initiative around the Federation of Enterprises of Congo (FEC), which is a non-profit organization under the terms of the law $n^{\circ} 004 / 2001$ of July 20, 2001 bearing general provisions applicable to non-profit associations and public utility establishments. This private institution allows Congolese entrepreneurs to meet and discuss issues that concern their activities and, where appropriate, defend their interests for the achievement of their individual or collective goals. The FEC has national, provincial and municipal bodies. Thus, it includes organs with general national competence which are the General Assembly; the Board of Directors; the Management Committee and the Auditors); but also organs with special national competence called Commissions or Chambers and organs with general material competence within the limits of their territorial jurisdiction.
\end{abstract}

* Licencié en Droit à l'Université de Kinshasa et chercheur en Droit public. marckmangaza@gmail.com. 


\section{Introduction}

Sur le plan juridique, la Fédération des entreprises du Congo (FEC) est régie par les dispositions de la Loi $n^{\circ}$ 004/2001 du 20juillet 2001 portant dispositions générales applicables aux associations sans but lucratif et aux établissements d'utilité publique, et par la Loin $^{\circ} 015 / 2002$ du 16 Octobre 2002 portant Code du Travail ainsi que par les dispositions de ses statuts ${ }^{1}$. La FEC constitue à la fois la chambre de commerce et d'industrie et la principale organisation patronale de la République démocratique du Congo. Ancienne Association nationale des entreprises du Zaïre (ANEZA), elle fut créée en 1972 de la fusion de la Fédération des Associations provinciales des entreprises du Zaïre (FERZA), la Fédération Nationale des Chambres de Commerce, de l'Industrie et d'Agriculture (FNCCIA) et l'Association pour la Promotion et la Défense des Intérêts des Commerçants Congolais (APRODECO).

Rebaptisée Fédération des entreprises du Congo lors du changement de nom du pays en mai 1997, elle représente actuellement plus de 2500 sociétés2 issues de tous les secteurs de l'économie congolaise. La FEC devrait envisager de fournir plus de services aux entreprises, en particulier, aux petites et moyennes entreprises aux problèmes pratiques desquelles les mécanismes du marché n'apportent pas spontanément des réponses. Son intervention est sous-dimensionnée au niveau de nombreux services qui concourent pourtant à la formalisation de l'économie, et donc à l'investissement et à la dynamique de diversification $^{2}$. De sa création à son développement, la vie d'une entreprise est jalonnée d'étapes délicates. Pour parer aux éventuelles périodes difficiles qu'elle peut rencontrer durant son évolution, la FEC améliore sans cesse ses prestations afin de créer un climat propice aux affaires. La FEC valorise également les ressources locales, cultive l'esprit d'entreprendre et dynamise l'image de la république démocratique du Congo et de ses entreprises. De nos jours, une entreprise se doit d'être innovante tant dans la qualité de ses services que de ses produits. La compétitivité actuelle doit absolument pousser leurs décideurs à se surpasser afin de se faire une place sure dans le marché qu'elle occupe. Pour les accompagner dans cette démarche à la recherche de l'innovation, la FEC réunit les meilleures compétences afin de leur apporter des solutions pertinentes afférentes à leurs métiers. La FEC guide pas à pas les entreprises vers leur réussite, elle est leur partenaire de tous les instants afin de «Bâtir ensemble » leurs projets.

\section{A. Mission et Objet de la FEC}

La Fédération a pour objet d'assumer les fonctions de Chambre de Commerce, d'industrie, de métiers, des mines, d'agriculture et d'autres secteurs ainsi que d'Organisation Profession-

1 Article 2, Loi $\mathrm{n}^{\circ} 004 / 2001$ du 20 juillet 2001, Portant dispositions générales applicables aux associations sans but lucratif et aux établissements d'utilité publique.

2 Capdeville J.-L., « La notion d'organe ou de représentant de la personne morale », Paris, 2018, pp.7 -12 . 
nelle des Employeurs. Pour ce faire, elle est notamment chargée de:1. Promouvoir les intérêts des entreprises de différents secteurs d'activités économique, sociale et scientifique, en harmonie avec le bien commun. A cette fin, elle est notamment chargée de : assumer la défense des intérêts des entreprises membres; coopérer à l'élaboration et à la mise en œuvre du programme de développement économique et social du pays; renforcer les relations commerciales fructueuses et l'entente entre les membres; susciter et consolider les rapports harmonieux entre les pouvoirs publics et la communauté des entreprises; poursuivre une action dynamique tendant à harmoniser la législation économique, commerciale et fiscale avec les intérêts des entreprises au bénéfice du développement économique; inciter, encourager et suivre dans tous les milieux une politique de financement et d'investissement rentable; informer tous les milieux aux fins d'une meilleure compréhension des phénomènes économiques en général et de la vie et des intérêts des entreprises en particulier; œuvrer pour l'établissement d'un climat favorable entre l'entreprise, ses travailleurs et leurs organisations syndicales; encourager et valoriser la formation professionnelle chez ses membres ainsi que celle de leur personnel; favoriser toute action de nature à promouvoir la formation et la recherche scientifique pour un développement intégré de la nation; œuvrer pour la bonne gouvernance et la lutte contre la corruption au sein des entreprises ${ }^{3}$. Représenter auprès des pouvoirs publics, des organismes nationaux, étrangers et internationaux les activités industrielles, minières, agricoles, commerciales, sociales, artisanales ainsi que les employeurs; Conseiller les entreprises dans l'interprétation des dispositions légales et réglementaires tant nationales qu'étrangères, collecter et diffuser celles-ci par les membres; Prendre des participations dans la gestion des entreprises d'intérêt général en vue de l'autofinancement de ses activités.

Peut devenir membre de la Fédération, toute personne physique commerçante ou morale régulièrement constituée en entreprise de droit privé ou public ou toute autre organisation structurée d'entreprises dûment revêtue de la personnalité juridique, qui exploite légalement sur le territoire de la République Démocratique du Congo une activité commerciale, industrielle, minière, agricole, artisanale, sociale ou libérale. Le dossier de demande d'affiliation est constitué d'une lettre de demande d'affiliation de l'impétrant, du bulletin d'adhésion dûment rempli, des preuves de la constitution régulière de l'entité (personne morale) ou de qualité de commerçant (personne physique) et de la preuve de parrainage d'un membre en ordre de cotisation. L'impétrant peut faire recours auprès du Conseil d'Administration en cas de refus de sa demande d'affiliation ${ }^{4}$. La qualité de membre se concrétise par le paiement d'une cotisation annuelle à la Fédération et la signature du Code d'éthique.

3 Voir Articles 40, 44, Fédération des entreprises du Congo (FEC), Statuts révises et coordonnes (Mars 2011).

4 Article 5, Fédération des entreprises du Congo (FEC), Statuts révises et coordonnes (Mars 2011). 


\section{B. L'organisation et fonctionnement de la FEC}

La Fédération comporte des organes à compétence nationale générale: l'Assemblée Générale; le Conseil d'Administration; le Comité de Direction; les Commissaires aux comptes. Des organes à compétence nationale spéciale dénommés Commissions ou Chambres : Commission de l'Economie, Finances et Budget; Commission Juridique; Commission des Relations Extérieures; Commission Sociale; Commission de l'Industrie; Commission Agriculture et Forêts; Commission de l'Energie; Commission des Transports; Commission des Télécommunications; Commission des Hydrocarbures; Commission du Commerce et Petites et Moyennes Entreprises, Commission des Travaux Publics, Chambre des Mines; Commission des Femmes Entrepreneurs; Commission des Sages. Des organes à compétence matérielle générale dans les limites de leur ressort territorial : l'Assemblée Provinciale; le Conseil Provincial; le Comité de District;le Comité de Territoire ou de Commune.

Le Conseil d'Administration peut créer des organes à compétence nationale spécialisée permanents ou temporaires auxquels il confie le soin d'étudier une ou plusieurs questions particulières intéressant la Fédération. Le Conseil d'Administration, sur une décision spéciale prise à la majorité absolue de ses membres, déterminera la répartition des Commissions Nationales, telles qu'énumérées à l'article 10 des Statut, et les attributions des Premiers Vice-Présidents et des Vice-Présidents. Le Conseil d'Administration peut créer un centre d'arbitrage autonome en vue du règlement des différends contractuels ou telles sections provinciales ou professionnelles qu'il juge utile à l'organisation de la Fédération et à la poursuite de son objet social. Il en détermine le ressort et la compétence matérielle. Le fonctionnement des sections provinciales est régi, mutatis mutandis, par les dispositions des articles 42 à 47 . Il en est de même du Centre d'Arbitrage ou de telle section que le Conseil d'Administration crée. Les organes à compétence nationale spéciale dépendent du Conseil d'Administration, elles lui font rapport. Les Présidents des organes à compétence nationale spéciale sont choisis au sein du Conseil d'Administration; leurs membres peuvent être choisis au sein ou en dehors du Conseil d'Administration. Le Président du Centre d'Arbitrage est choisi par le Conseil d'Administration, au sein ou en dehors de celui-ci.

L'Assemblée Générale ne délibère que sur les points inscrits à son ordre du jour. L'Assemblée Générale Ordinaire ne peut délibérer valablement qu'à la moitié de l'ensemble des voix reconnues aux membres de la Fédération présents ou représentés. Il en est de même des résolutions de l'Assemblée Générale Extraordinaire, sauf en cas de modifications des statuts ou de dissolution de la Fédération. En cas de modification statutaire ou de dissolution de la Fédération, les résolutions sont prises au deux tiers au moins des voix reconnues à l'ensemble des membres de la Fédération présents ou représentés. Si lors de la première convocation, l'Assemblée Générale Ordinaire ou Extraordinaire, selon le cas, ne réunit pas le quorum de voix exigées pour le vote des résolutions, une seconde Assemblée Générale est convoquée avec le même ordre du jour ${ }^{5}$. Cette seconde Assemblée

5 Article 14, Fédération des entreprises du Congo (FEC), Statuts révises et coordonnes (Mars 2011). 
Générale délibère valablement, quel que soit le nombre des membres effectifs présents ou représentés. Les résolutions ou décisions valablement prises lors d'une Assemblée Générale engagent chaque membre de la Fédération. L'Assemblée Générale se réunit en session extraordinaire en un lieu fixé par le Comité de Direction ou le Conseil d'Administration chaque fois que ce dernier le juge nécessaire ou chaque fois que la demande en est faiteau Président du Conseil d'Administration soit par un tiers au moins des Conseils Provinciaux, soit à l'initiative des membres réunissant un tiers des voix; une telle demande, dans tous les cas, doit être adressée au

Président du Conseil d'Administration et suffisamment motivée en indiquant l'ordre du jour demandé. L'Assemblée Générale Extraordinaire est convoquée par le Président du Conseil d'Administration dans les formes et délais prescrits. Ses résolutions sont prises à la majorité simple des voix présentes ou représentées, sauf en cas de modification des statuts ou de dissolution de la Fédération.

Le Conseil d'Administration est l'organe de gestion de la Fédération. Il dispose, en dehors des sessions de l'Assemblée Générale, de tous pouvoirs, et prend toute décision de nature à promouvoir l'objet de la Fédération tel que défini. A cet effet, il est habilité, à accomplir tous les actes de la vie juridique, et notamment emprunter, ester en justice, accepter dons et legs, posséder ou acquérir des immeubles et tous les biens nécessaires à la réalisation de son objet social et ce, par le biais de son Président. Le pouvoir de signer les actes engageant la Fédération, toutes délégations de pouvoirs, générales ou spéciales, et toutes procurations sont du ressort du Conseil d'Administration qui peut, au travers de son Président, les conférer à toute personne mandatée dans l'intérêt de la Fédération. Les membres du Conseil d'Administration sont nommément élus parmi les personnes physiques représentant les entreprises membres, en fonction de leur intérêt aux activités de la Fédération, de leurs hautes qualités morales et compétences personnelles ${ }^{6}$. Toute fois, à la demande de l'entreprise membre, ils peuvent être remplacés automatiquement par le Chef de l'Entreprise.

Le Conseil d'Administration est un collège de membres de la Fédération, désignés de la manière suivante : les Présidents élus des Conseils Provinciaux; les membres dont la contribution au budget de la Fédération atteint le plafond des cotisations; six membres respectifs pour chacune de trois autres catégories déterminées en fonction de tranches de cotisations; quatre membres des chambres de commerce bilatérales affiliées à la Fédération, en règle de cotisation et élus par leurs pairs; les anciens Présidents Nationaux de la Fédération; l'Administrateur Délégué. Les membres cités aux points 3 et 4 de cette disposition sont élus au sein de leur groupe lors de la tenue de l'Assemblée Ordinaire par l'ensemble des entreprises de leur catégorie, constituées en collège électoral. Le collège électoral veille à assurer au sein du Conseil d'Administration une représentation équilibrée des branches d'activités de la catégorie. Les membres du Conseil d'Administration élisent en leur sein tous les trois ans, à l'issue de l'Assemblée Générale Ordinaire et conformément au règlement 
intérieur le régissant, un Président du Conseil d'Administration pour un mandat de trois ans renouvelable autant de fois que nécessaire. Sur proposition du Président élu, les membres du Conseil d'Administration désignent dans les mêmes conditions les Vice-Présidents. Le Président peut être déchargé de sa fonction, à la demande de la Commission des sages, par vote du Conseil d'Administration.

L'Administrateur Délégué exécute les décisions et recommandations du Conseil d'Administration. Il est guidé dans cette exécution et dans la gestion journalière par le Comité de Direction. L'Administrateur Délégué établit chaque année le rapport annuel d'activités du Conseil et les comptes de la Fédération destiné à l'Assemblée Générale, et les soumet au Conseil d'Administration, après avis du Comité de Direction. Il dresse de même le budget prévisionnel des dépenses et des recettes et le présente au plus tard le premier octobre de chaque année au

Conseil d'Administration, après avis du Comité de Direction. Les comptes de la Fédération font l'objet d'un règlement financier.

\section{Conclusion}

Cette étude, dont l'objet a été de présenter et d'analyser le cadre juridique, l'organisation et le fonctionnement de la FEC en République démocratique du Congo, permet de situer avec précision la place de la FEC dans le système économique de la RDC. La FEC est une Association sans but lucratif puisse que celle-ci ne se livre pas à des opérations industrielles ou commerciales et ne cherche pas à procurer à ses membres un gain matériel quelconque. D'où elle est une asbl de nature économique. La FEC comprendre des organes à compétence nationale générale (1. l'Assemblée Générale; 2. le Conseil d'Administration; 3. le Comité de Direction;4. les Commissaires aux comptes), des organes à compétence nationale spéciale dénommés Commissions ou Chambres actuellement au nombre de 15 et des organes à compétence matérielle générale dans les limites de leur ressort territorial qui sont l'Assemblée Provinciale; le Conseil Provincial; le Comité de District; le Comité de Territoire ou de Commune.

Sur le plan économique cette asbl joue un rôle très important dans la mesure où elle permet de centraliser de manière objective et au profit des potentiels investisseurs ou entrepreneurs les informations relatives à l'environnement du monde des affaires au Congo. Etant un fait privé, l'intervention de l'Etat un est très minimale, plus souvent limité à l'ordre public et les bonnes mœurs. C'est dans ce contexte que la FEC fonctionne en regroupant les investisseurs et les entrepreneurs du pays.

\section{Bibliographie}

\section{Textes juridiques}

Loi n 004/2001 du 20 juillet 2001, Portant dispositions générales applicables aux associations sans but lucratif et aux établissements d'utilité publique. 
Fédération des entreprises du Congo (FEC), Statuts révises et coordonnes (Mars 2011).

Constitution de la République Démocratique du Congo telle que modifiée par la loi n¹1/002 du 20 janvier 2021 portant révision de certains articles de la constitution de la République Démocratique du Congo du 18 février 2006, in JORDC, $52^{\text {ème }}$ année, numéro spécial, Kinshasa- 5 février 2011.

La loi n015-2002 portant code du travail telle que modifiée et complétée par la loi n¹6/010 du 15 juillet 2016, in JORDC, numéro spécial du 16 octobre 2002.

\section{Ouvrages et articles}

Andre brun et Henri Galland, Droit du travail, 2ème éd., Tome I, Sirey 1978.

Luc Nganda Fumabo, l'audience judicaire, 2 èmeéd. RIVAGES AFRICAINS, Kinshasa.

Botakile Batanga, précis du contentieux administratif congolais, T1 éd. Academia Louvain-la-Neuve, 2018.

FEC, Syndicat patronal, disponible sur http://www.fec-rdc.com/index.php/nos-services/syndicat-patron al, consulté le 21 aout 2021.

Capdeville J.-L., « La notion d'organe ou de représentant de la personne morale », AJ Pénal, 2018.

Paillusseau J., « Le droit moderne de la personnalité morale », RTD Civ. 1993.

\section{Autres documents}

Marc Atibu Saleh Mwekee, Recueil des formalités et obligations des entreprises en matière du travail et de la sécurité sociale, Kinshasa, mai 2014 